\title{
Pulmonary embolism: the diagnosis, risk-stratification, treatment and disposition of emergency department patients
}

\section{Daniel Corrigan, Christiana Prucnal, Christopher Kabrhel}

Center for Vascular Emergencies, Department of Emergency Medicine, Massachusetts General Hospital, Harvard Medical School, Boston, MA, USA

The diagnosis or exclusion of pulmonary embolism (PE) remains challenging for emergency physicians. Symptoms can be vague or non-existent, and the clinical presentation shares features with many other common diagnoses. Diagnostic testing is complicated, as biomarkers, like the D-dimer, are frequently false positive, and imaging, like computed tomography pulmonary angiography, carries risks of radiation and contrast dye exposure. It is therefore incumbent on emergency physicians to be both vigilant and thoughtful about this diagnosis. In recent years, several advances in treatment have also emerged. Novel, direct-acting oral anticoagulants make the outpatient treatment of low risk PE easier than before. However, the spectrum of PE severity varies widely, so emergency physicians must be able to risk-stratify patients to ensure the appropriate disposition. Finally, PE response teams have been developed to facilitate rapid access to advanced therapies (e.g., catheter directed thrombolysis) for patients with high-risk PE. This review will discuss the clinical challenges of PE diagnosis, risk stratification and treatment that emergency physicians face every day.

Keywords Pulmonary embolism; Venous thrombosis; Risk stratification; Thrombolysis; Hospital rapid response team
eISSN: $2383-4625$

Received: 6 May 2016

Revised: 25 May 2016

Accepted: 25 May 2016

Correspondence to: Christopher Kabrhel Center for Vascular Emergencies, Department of Emergency Medicine, Massachusetts General Hospital, Zero Emerson Place, Suite 3B, Boston, MA 02114, USA

E-mail: ckabrhel@partners.org

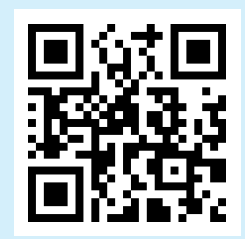

How to cite this article:

Corrigan D, Prucnal C, Kabrhel C. Pulmonary embolism: the diagnosis, risk-stratification, treatment and disposition of emergency department patients. Clin Exp Emerg Med 2016;3(3):117-125.

This is an Open Access article distributed under the terms of the Creative Commons Attribution Non-Commercial License (http:// creativecommons.org/licenses/by-nc/4.0/). 


\section{INTRODUCTION}

Pulmonary embolism (PE) is the third most common cause of cardiovascular death among Americans, behind myocardial infarction and stroke. ${ }^{1}$ Mortality varies greatly, depending on various factors including age, comorbid conditions, and stability on presentation. Patients with low-risk PE have a 1-year survival rate over 95\%. In contrast, patients presenting with high risk PE and hemodynamic instability have an approximately $40 \%$ mortality rate within 90-days. ${ }^{2}$ In this review, we will discuss the basic pathophysiology of $\mathrm{PE}$, risk factors for developing $\mathrm{PE}$, and standard diagnostic testing modalities. We will also cover risk stratification of patients presenting with PE and the implications for treatment and disposition.

\section{PATHOPHYSIOLOGY}

PE occurs when clots formed in the deep venous system dislodge or break loose, travel through the heart, and become lodged in the pulmonary vasculature. While small PE can frequently lyse spontaneously, larger PE can cause a sudden and persistent rise in pulmonary artery pressure, which can lead to circulatory collapse.

Clot formation usually begins at sites of tissue or vascular trauma where recruited activated monocytes expose blood to tissue factor on their surfaces and overwhelm the body's natural anticoagulant and fibrinolytic mechanisms. These deep venous thrombi (DVT), composed mainly of red blood cells, platelets, and fibrin, tend to form in the venous sinuses or cusps of the lower extremity deep veins. While the main concern is that part or all of a DVT will embolize to the pulmonary vasculature, the vascular congestion caused by DVT also has multiple effects on the limb. There is often associated swelling and discomfort, as well as eventual loss of valve competency. This allows for lack of forward flow causing more swelling, stasis, and thrombus propagation.

A longer-term complication is "post-thrombotic syndrome" which can result if these changes persist over time. Post-thrombotic syndrome consists of a constellation of symptoms including calf pain, swelling, and stasis skin changes, especially hyperpigmentation at the medial malleolus. It affects approximately half of patients who suffer DVT, although the onset can be delayed several years following DVT formation. ${ }^{3}$

Once a clot becomes lodged in the pulmonary vasculature, it causes obstruction. The extent of this obstruction, combined with the presence of any underlying cardiopulmonary disease are the most important factors in determining whether right ventricular dysfunction will develop. ${ }^{4}$ The blockage, combined with the release of vasoactive mediators, causes a sudden rise in pulmonary artery pressure, which increases right ventricular afterload. The right ventricle, a thin-walled structure compared to the robust left ventricle, can dilate and become hypokinetic in response to the rapid rise in afterload. Right ventricular dilatation can ultimately lead to under-filling of the left ventricle as the interventricular septum protrudes into the left ventricle, decreasing cardiac output and coronary perfusion which, in turn, can lead to eventual circulatory collapse. ${ }^{4}$

\section{RISK FACTORS FOR DVT AND PE}

There are multiple factors that can increase one's chance of developing DVT and PE. These factors can be inherited, acquired over time, or provoked. Various inherited conditions increase a patient's risk for venous thromboembolism (VTE), the most common of which are factor V Leiden and prothrombin gene mutation (G20210A) with population prevalences of approximately $4 \%$ to $5 \%$ and $2 \%$ to $4 \%$, respectively., ${ }^{5,6}$ patients with factor $V$ Leiden, the increased risk of VTE is 2- to 7-fold in heterozygous individuals and up to 40 -fold in those who are homozygous.?

The most common acquired risk factors for VTE include: increasing age, venous insufficiency, obesity, smoking, rheumatologic conditions, cardiovascular disease, previous VTE, and antiphospholipid antibody syndrome. The mechanism behind increasing age appears to be that naturally circulating anticoagulants (protein $\mathrm{C}$ and protein $\mathrm{S}$ ) decrease more than procoagulation factors over time, creating an increased prothrombotic state. This, combined with increased venous stasis in the lower extremities, increases the rates of DVT and PE in the elderly population. Obesity is a known risk factor for VTE, and data from the Nurses' Health Study found that, among the most obese subjects (body mass index $>35$ ), there was a 6 -fold increase in risk when compared to normal-weight subjects. ${ }^{8}$ This same study showed that hypertension and cigarette smoking were also associated with increased risk of idiopathic $\mathrm{PE} \mathrm{P}^{9,10}$

Provoking risk factors are those that create a significant increase in VTE risk that can resolve once the provoking factor is eliminated. Provoking factors typically increase the risk of VTE more than other risk factors. Common provoking factors include: cancer, exogenous hormone use (in particular, recent initiation of estrogen-containing formulations), pregnancy/postpartum state, limb immobility, recent trauma or surgical procedure, and indwelling catheters. In a national registry, the incidence of DVT and PE in patients with cancer was twice that of patients without cancer. ${ }^{11}$ Cancer is considered a provoking factor because the risk of VTE is elevated in patients with active cancer, but a history of treated cancer in remission is not associated with increased VTE 
risk. Exogenous hormone use has long been known to be associated with increased risk of VTE. In patients taking oral contraceptives there is a 3- to 4-fold increased risk of VTE, and those using "third-generation" progesterone formulations are especially at risk. ${ }^{12}$ The increased risk of VTE during pregnancy starts in the first trimester and continues through the postpartum period, with the greatest risk during the first 2 weeks postpartum. ${ }^{13}$ The increased risk of VTE associated with prolonged limb immobility typically occurs after about 72 hours of acute limb immobility. ${ }^{14}$ It is also important to note that provoking factors can interact with other risk factors to increase VTE likelihood more than either factor would individually. ${ }^{15}$

\section{INITIATING THE DIAGNOSTIC WORKUP: PRE- TEST PROBABILITY ASSESSMENT}

The first step in the diagnostic workup for PE and DVT is determining the patient's pre-test probability of the diagnosis. The patient's pre-test probability determines whether testing is indicated at all, and which tests should be performed. While multiple prediction rules for PE exist, the two most commonly used are the Wells score and the revised Geneva score for PE. The Wells score (Table 1) consists of 6 objective questions and one subjective question asking whether "PE is the most likely diagnosis." Based on the score (points are awarded for each positive answer) pre-test probability of PE may be assigned as either low ( $<2$ points), intermediate (2 to 6), or high (>6). Alternatively, the Wells score can be dichotomized into low probability (less than or equal to 4 points) and high probability ( $>4)$, which some argue is more easily applied to clinical decision-making. ${ }^{16}$

The revised Geneva score (Table 2) consists entirely of objective questions. Zero to 3 points is considered low probability, 4 to 10 points is intermediate probability, and more than 11 points is considered high probability of PE. Both scores have been validated

Table 1. Wells score for PE

\begin{tabular}{|c|c|c|}
\hline \multicolumn{2}{|l|}{ Variable } & Points \\
\hline \multicolumn{2}{|l|}{ Previous PE or DVT } & +1.5 \\
\hline \multicolumn{2}{|c|}{ Heart rate $>100 \mathrm{bpm}$} & +1.5 \\
\hline \multicolumn{2}{|c|}{ Recent surgery or immobilization } & +1.5 \\
\hline \multicolumn{2}{|l|}{ Clinical signs of DVT } & +3 \\
\hline \multicolumn{2}{|l|}{ Hemoptysis } & +1 \\
\hline \multicolumn{2}{|l|}{ Cancer } & +1 \\
\hline \multicolumn{2}{|c|}{ Alternative diagnosis less likely than PE } & +3 \\
\hline Probability of PE & Score & Prevalence of PE \\
\hline Low & $\leq 4$ & $7.8 \%$ \\
\hline High & $>6$ & $61 \%$ \\
\hline
\end{tabular}

$\mathrm{PE}$, pulmonary embolism; DVT, deep venous thrombi. and perform similarly in clinical practice. However, studies also show that clinical gestalt assessed by an experienced clinician is just as accurate as these formal decision rules. ${ }^{16}$

The pulmonary embolism rule-out criteria (PERC) was designed to identify patients in whom the risk of testing outweighs the benefits (the "test threshold", which for PE was calculated as a $2 \%$ prevalence). The PERC consists of eight objective variables that can be applied to patients with low clinical (pre-test) probability of PE (Table 3 ). ${ }^{17}$ The sensitivity of the PERC rule is $96 \%$ to $100 \%$ and specificity is $15 \%$ to $27 \%$. In the initial derivation and validation study of low and very-low probability patients, the prevalence of PE in patients that were PERC negative was $<1.5 \%{ }^{17}$ The PERC was subsequently validated in an NIH-funded multicenter study of 8,138 patients. Twenty percent $(n=1,666)$ of enrolled patients had a low clinical probability of PE and were PERC negative. ${ }^{18}$ Of this group, 15 patients were positive for VTE within 45 days of initial presentation-a false negative rate of $<1 \%$, with an upper confidence limit of $<2 \%$. The sensitivity was $97.4 \%$ and the specificity was $21.9 \%$. The authors therefore concluded that a low pretest probability and a negative PERC effectively rules out $P E$ with a false negative rate lower than the test-threshold. The PERC rule has now been incorporated into guidelines for the diagnosis of PE. ${ }^{18,19}$

Table 2. Revised Geneva score for PE

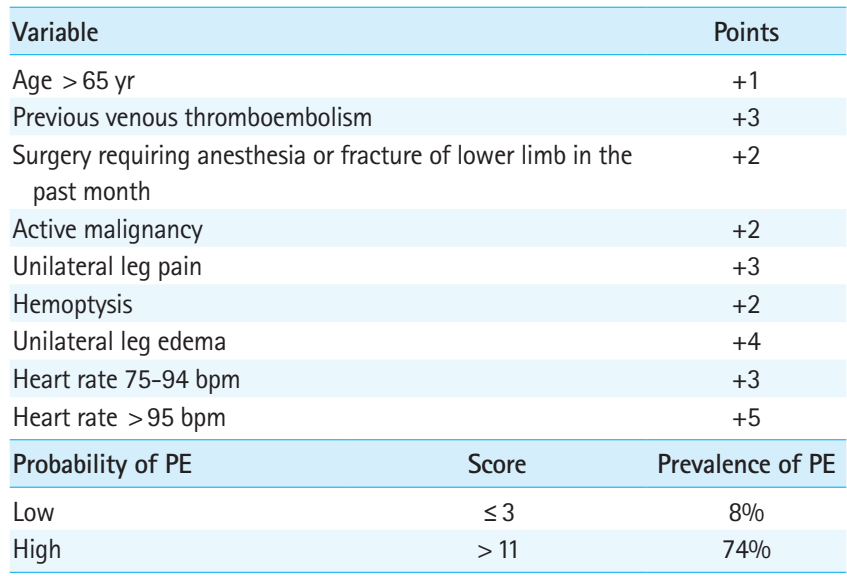

$\mathrm{PE}$, pulmonary embolism.

Table 3. Pulmonary embolism rule-out criteria

\section{Variable}

Age $<50$ yr

Pulse $<100 \mathrm{bpm}$

$\mathrm{SaO}_{2}>94 \%$

No unilateral leg swelling

No hemoptysis

No recent trauma or surgery

No prior pulmonary embolism/ deep venous thrombi

No hormone use 


\section{TESTING AND DIAGNOSIS}

\section{DVT diagnosis}

The diagnostic test of choice for detection of proximal DVT is venous ultrasonography, with loss of vein compressibility being diagnostic of DVT. This modality is readily available in the emergency department at all times and poses little to no risk to the patient. A meta-analysis assessing emergency physicians' ability to diagnose DVT on ultrasound confirmed that emergency physicians' weighted mean sensitivity and specificity for DVT were on par with radiology-performed studies (96.1\% and 96.8\% respectively). ${ }^{20}$ Since approximately half of patients with PE have no imaging evidence of DVT, a negative venous ultrasound is not sufficient to rule out PE. However, evidence of DVT on ultrasound along with signs and symptoms of PE is considered diagnostic, and sufficient for initiation of treatment. Accordingly, analyses suggest that a non-invasive strategy combining ultrasound and D-dimer testing (below) as the first diagnostic tests for PE may be the most cost-effective approach. ${ }^{21}$

For most patients in whom VTE is a concern, the most appropriate first test is usually the D-dimer. This test analyzes blood for a breakdown product of cross-linked fibrin that is detectable when plasmin breaks down organized clot. The test is highly sensitive for VTE (95\% sensitive when using the Food and Drug Administration (FDA)-approved cutoff (typically $<500 \mathrm{ng} / \mathrm{mL}$ ). A negative D-dimer effectively rules out $\mathrm{PE}$, with post-test probability $<2 \%$, in patients with non-high pre-test probability. ${ }^{22}$ However, D-dimer assays are poorly specific (typically $40 \%$ to $50 \%$ ), so further testing is needed to rule out VTE in a large proportion of patients who have a positive D-dimer. D-dimer results are even more likely to be elevated in certain states, so the usefulness of the test is limited in the elderly ( $>80$ years), pregnant women (especially in the third trimester), patients with sickle cell anemia, and those with major trauma or surgery in the past month. ${ }^{22,23}$ There has been some research into utilizing adjusted D-dimer values according to age, in which the cut off of a positive D-dimer for patients over 50 years was calculated as: age multiplied by 10 . This has been evaluated prospectively and found be associated with low risk of clinically significant PE or death, however more research is still needed to validate this adjustment for incorporation into everyday practice. ${ }^{23}$

\section{PE diagnosis}

The standard imaging modality for diagnosis of PE is contrast enhanced computed tomography pulmonary angiography (CTPA). Modern computed tomography scanners provide excellent resolution and enable the detection of smaller thrombi than previ- ously possible. PE are visualized as filling defects (gray) in contrast-filled (white) pulmonary arteries and most often arise at sites of bifurcation or vessel narrowing. One of the largest diagnostic studies of CTPA for PE was the landmark PIOPED (Prospective Investigation of Pulmonary Embolism Diagnosis)-II study, which showed the sensitivity of CTPA was $83 \%$ to $90 \%$ depending on the location of the PE and whether computed tomographic venography of the lower extremities was included. ${ }^{24}$ CTPA was found to have specificity $>95 \%$ in this study. Subsequent outcomes studies confirmed that CTPA can safely rule out PE in patients with a high pre-test probability of PE or a positive D-dimer with fewer than $2 \%$ of patients diagnosed with PE in the three months after a negative CTPA. ${ }^{25}$

An alternative to CTPA is ventilation/perfusion lung scanning, which is particularly useful for patients in whom CTPA is contraindicated, such as: patients with chronic kidney disease, contrast dye allergy, or radiation concerns in pregnancy. This involves a radionucleotide perfusion scan and a ventilation scan. If the perfusion scan is normal (i.e., shows no perfusion defects), this effectively rules out PE and the ventilation portion of the scan is unnecessary. When the perfusion scan is abnormal, the ventilation portion of the scan must be performed. A minimum of two wedgeshaped defects in a segmental or larger vascular distribution along with signs of normal ventilation in the same segments defines a high-probability scan, which is diagnostic of PE. Unfortunately, as many as two thirds of patients will have a non-diagnostic scan, which is neither normal nor high-probability. This is the main reason that ventilation/perfusion scanning was supplanted by CTPA as the first imaging test for PE.

Patients with severe PE can present with significant hemodynamic instability, making diagnosing PE with imaging difficult. Patients with large PE are preload dependent due to impaired left ventricular filling, thus interventions to increase preload, like boluses of isotonic intravenous fluid, are recommended, while interventions that decrease preload, like positive pressure ventilation and diuresis may be harmful and should be avoided if possible. In patients with high probability of PE who are unable to obtain definitive diagnostic imaging (e.g., CTPA) within 2 hours, guidelines recommend empiric anticoagulation. ${ }^{26}$ Similarly, in patients with intermediate probability of PE empiric anticoagulation is recommended in cases where diagnostic imaging will take longer than 24 hours. ${ }^{26}$

\section{RISK STRATIFICATION}

The severity of PE varies widely. Patients with PE may be asymptomatic or may present with complete cardiovascular collapse. For low-risk $\mathrm{PE}$, initiation of anticoagulation and discharge home 
may be appropriate, while for high-risk PE, thrombolysis, surgical thromboembolectomy and admission to the intensive care unit may be necessary. Risk stratification after PE diagnosis is therefore of paramount importance.

The main determinants of PE severity are: presence of right heart strain either on echocardiography or computed tomography, myocardial damage based on troponin elevation, and overall clot burden. Although the overall clot burden appears to have only a variable relationship to outcomes, large PE are typically associated with worse outcomes than smaller or segmental PE. ${ }^{27}$ One must also consider the general overall health of the patient and take into account age and presence of comorbid illness that may affect prognosis. While there is variability in the classifications systems used to define short-term risks from PE, for the purposes of this discussion we will categorize patients as low-risk, intermediate-risk, and high-risk. ${ }^{28,29}$

\section{High risk}

Patients with PE who present with hypotension, syncope, bradycardia, or the inability to maintain adequate oxygenation are at risk for sudden death, even with appropriate treatment. Patients with large PE and residual clot present in the heart ("clot-in-transit") or iliofemoral veins should also be considered at high risk for decompensation. High-risk patients often require emergent intervention and admission to the intensive care unit.

\section{Intermediate risk}

Patients who presents with end organ damage but are hemodynamically stable can be considered intermediate risk. Right heart strain on echocardiography, in the setting of a large $P E$, is also associated with an increased risk of clinical deterioration and/or short-term death. ${ }^{28}$ Other factors, such as an elevated troponin indicating cardiac ischemia, altered mental status, and the presence of co-morbid illness have also been correlated with early clinical deterioration and higher incidence of short-term death. ${ }^{28,30}$

\section{Low risk}

Patients found to have PE without evidence of end organ damage or hemodynamic instability may be able to be safely discharged early after initiation of anticoagulation. In an analysis of 298 consecutive patients with $\mathrm{PE}$, we found that $2 / 3$ of patients suffered no clinical deterioration and required no hospital-based interventions after PE. This suggests that a large proportion of patients derive no benefit from hospitalization after $\mathrm{PE}$, and may be safe for discharge from the ED. In our analysis, similar to a prospective study by Sanchez et al. ${ }_{1}^{28}$ hypotension, hypoxia, history of coronary artery disease, evidence of right heart strain, and re- sidual DVT were all found to be independently associated with clinical deterioration. Patients who present with none of these risk factors are likely at low risk for early clinical deterioration and are ideal candidates for early discharge after initiation of anticoagulation. ${ }^{29,31}$

\section{TREATMENT AND DISPOSITION}

For all patients with PE who do not have a significant contraindication, anticoagulation treatment is essential in halting the propagation of thromboembolism. There is now a wide range of anticoagulation options and other interventions available depending on a patient's initial presentation, risk of early clinical deterioration, and mortality. For most patients, in particular low risk patients, treatment with a long acting oral or subcutaneous anticoagulant is indicated. However, for some patients, in particular those likely to undergo an intervention or thrombolysis or those at high risk of bleeding, the ability to rapidly "turn off" anticoagulation may be beneficial. In these cases, unfractionated heparin (UFH) is the anticoagulant of choice.

\section{Anticoagulation}

Anticoagulation does not dissolve clot that already exists, but it decreases new thrombus formation while permitting the body's natural fibrinolytic mechanisms to lyse existing clot. For patients with acute $P E$ and no active cancer, guidelines recommend initiation of a direct acting oral anticoagulant (DOAC): apixaban, dabigatran, edoxaban or rivaroxaban. ${ }^{26}$ Of these, apixaban and rivaroxaban do not require 'bridging' therapy with heparin, so are better suited for initiation of therapy in ED patients. Compared with long-term warfarin therapy, these DOAC agents are associated with similar rates of recurrent PE but slightly lower rates of treatment-associated hemorrhage, in particular, intracranial hemorrhage. DOACs are administered orally, provide rapid onset of action, and do not require routine laboratory coagulation monitoring. These medications have few interactions and are generally associated with lower rates of major bleeding, making them preferable to warfarin. ${ }^{32}$

Currently, four DOACs have received US FDA approval for the treatment of VTE. Distinctions between the four US FDA approved DOACs must be considered when determining treatment recommendations. Dabigatran, is a direct thrombin (factor II) inhibitor approved for the treatment of acute VTE after initial treatment with heparin. ${ }^{17}$ Apixaban, edoxaban, and rivaroxaban, are factor Xa inhibitors and approved for treatment of VTE. However, while apixaban and rivaroxaban are approved as monotheraphy, edoxaban requires a heparin bridge similar to dabigatran. The initiation 
dose of apixaban is $10 \mathrm{mg}$ twice daily for 7 days followed by 5 $\mathrm{mg}$ twice daily after that period. The initiation dose of rivaroxaban is $15 \mathrm{mg}$ orally, twice daily, followed by $20 \mathrm{mg}$ daily after 21 days. All of the DOACs should be used with caution in patients with severe liver or kidney disease, and studies of high-risk subgroups of patients (e.g., antiphospholipid antibody) are limited.

None of the DOACs have been extensively tested in patients with active cancer, so low molecular weight heparin (LMWH) is still recommended as initial therapy for these patients. Trials are ongoing to assess the efficacy and safety of the DOACs in this population.

As above, low risk patients can often be discharged from the $E D$, an emergency department observation unit, or inpatient floor within 24 hours of diagnosis. Close follow-up and strict return instructions are required. However, the oral bioavailability of DOACs and the associated ability to initiate treatment without intravenous or subcutaneous injections (with apixaban or rivaroxaban) may help facilitate the outpatient treatment of PE and DVT.

The main clinical concern about the DOAC agents has been the lack of an effective reversal agent. However, this concern is largely mitigated by the lower risk of major and intracranial bleeding associated with the DOACs. In addition, idarucizumab, a monoclonal antibody designed for the reversal of anticoagulant effects of dabigatran, was recently approved by the US FDA. Studies of andexanet alfa, a recombinant protein designed to reverse the anticoagulant activity of direct and indirect factor Xa inhibitors is currently being studied and may be approved for use in the near future.

Heparins bind to antithrombin, thus increasing its activity and markedly accelerating inactivation of thrombin, factor $\mathrm{Xa}$, and factor IXa. Both unfractionated intravenous heparin and LMWH are effective for the treatment of acute PE. In patients with normal hepatic function and no history of heparin induced thrombocytopenia (HIT), UFH can be initiated with an IV bolus of $80 \mathrm{U} / \mathrm{kg}$, followed by a continuous infusion at $18 \mathrm{U} / \mathrm{kg} / \mathrm{hr}$, with a target activated partial thromboplastin time between 1.5 and 2.5 times the control value ( 60 to 80 seconds). The short half-life of UFH is advantageous for patients who may require embolectomy or thrombolysis or who are at high risk for bleeding (e.g., recent high risk surgery).

LMWHs is composed of small fragments of heparin that bind strongly to antithrombin working in the same way as heparin, but with improved bioavailability, a longer half-life, dose dependent clearance, and lower rates of HIT. Thus LWMH can be administered once or twice daily without the need for laboratory monitoring. The most commonly used LMWHs include enoxaparin, at a dose of $1 \mathrm{mg} / \mathrm{kg}$ twice daily; dalteparin, at a dose of $200 \mathrm{U} / \mathrm{kg}$ daily or 100 to $125 \mathrm{U} / \mathrm{kg}$ twice daily; and tinzeparin, at a dose of $175 \mathrm{U} / \mathrm{kg}$ daily. LMWHs are associated with a slightly lower rate of anticoagulation associated bleeding than UFH, with a comparable risk of recurrent PE. Thus, for patients who do not require thrombolysis or an invasive procedure, LMWHs are generally preferred over UFH. The subcutaneous administration route for LMWHs offers the possibility of treatment at home and thus allows for earlier hospital discharge. ${ }^{33}$

LMWH is also preferred over warfarin for patients with VTE and active cancer. In a randomized trial, monotherapy with dalteparin was shown to reduce VTE recurrence compared to long-term warfarin therapy, with $9 \%$ of dalteparin patients suffering a recurrence compared to $17 \%$ of warfarin patients. ${ }^{33} \mathrm{LMWH}$ is also preferred treatment in pregnancy, as warfarin is teratogenic and contraindicated.

For initial treatment of PE in patients with known HIT, fondaparinux, an anticoagulant that binds antithrombin and inhibits factor Xa is another alternative to the DOACs. Fondaparinux had no known effect on platelet function and does not cross-react with heparin-induced antibodies. Dosing is weight-based, from 5 to $10 \mathrm{mg}$ daily as a subcutaneous injection. Lepirudin and argatroban are intravenous direct thrombin inhibitors that bind directly to fibrin-associated thrombin or fibrin degradation products.

Warfarin, now a second line option for the long-term treatment of $P E$, produces hemostatically defective vitamin K-dependent coagulant proteins (factors II, VII, IX, and X). The anticoagulant response is variable among patients and thus frequent monitoring of the patient's prothrombin time/international normalized ratio is required to maintain it within the therapeutic window of 2 to 3 . Warfarin interacts with multiple other drugs and with vitamin K-containing foods and requires heparin bridging.

\section{Treatment of high-risk patients}

While the mainstay of treatment for all patients presenting with $P E$ is anticoagulation, high-risk patients may require additional treatment measures to reduce the clot burden. Systemic intravenous thrombolysis has been shown to improve mortality and hemodynamic stability when used for patients with high risk (massive) and high-intermediate risk (submassive) PE. ${ }^{32}$ However, systemic thrombolysis is associated with a high risk of hemorrhage. Thus, its use should be limited to high-risk, hemodynamically unstable PE patients and select intermediate-risk PE patients with a low bleeding risk.

Alteplase (t-PA) and tenecteplase are two thrombolytic agents that can be used in treatment of PE. Only alteplase is US FDA approved for the treatment of massive PE. Due to its short half-life of 4 to 6 minutes, alteplase requires a continuous IV infusion. An 
initial bolus of $15 \mathrm{mg}$ followed by an additional $85 \mathrm{mg}$ over 2 hours is standard dosing. For hemodynamically unstable patients, this relatively lengthy 2 hours infusion may be too slow and impractical. Here tenecteplase offers an alternative, with a longer 20 to 24 minutes half-life that is delivered in a single, weight-based bolus dose. Tenecteplase, however, is not currently FDA approved for VTE treatment, although it was used in two major randomized trials of PE thrombolysis. ${ }^{34,35}$

Catheter-directed thrombolysis may attenuate the bleeding risk associated with systemic thrombolysis. Catheter-directed thrombolysis involves catheter placement adjacent to the $P E$, with local infusion of thrombolytics into affected pulmonary arteries. Mechanical and ultrasonic clot disruption can be used to assist in thrombolysis. This targeted technique offers the benefit of both local delivery and lower drug dose (typically 10 to $20 \mathrm{mg}$ t-PA infused over 12 to 24 hours). In addition, the presence of catheters allows for PA pressure monitoring which can be used to guide discontinuation of therapy. ${ }^{36}$ As this procedure is relatively new, robust outcome data on patients treated with catheter-directed thrombolysis are lacking, with only one small randomized trial published. However, larger registry studies and meta-analyses of case series demonstrate clinical effectiveness and minimal bleeding risk associated with catheter directed thrombolysis. ${ }^{36,37}$

Surgical thromboembolectomy, performed via a median sternotomy can be effective for select patients with large PE, especially when performed at experienced centers. ${ }^{38}$ Historically, this procedure has typically been reserved for those with massive, hemodynamically unstable PE who either had an absolute contraindication or failed thrombolysis. Mortality has improved in recent years and is better if performed prior to the development of cardiogenic shock and end organ damage, but is still around 20\% likely due to the severe illness of patients referred for surgery. ${ }^{38,39}$

Highly specialized centers can also perform percutaneous suction thromboembolectomy. This involves cannulation of the venous system in two places for aspiration of the clot and reinfusion of blood following filtration and is typically reserved for patients with large proximal (e.g., saddle) emboli or clot-in-transit. 36,37

\section{PE response 'PE response teams'}

Some large academic centers have created multidisciplinary PE response teams (PERTs). Modeled on other rapid response teams, PERTs meet in real-time to discuss optimal therapy for patients presenting with life threatening PE. These multidisciplinary teams usually involve specialists in cardiology, cardiovascular surgery, emergency medicine, hematology, interventional radiology, pulmonary/critical care, vascular medicine and surgery. ${ }^{39}$ PERTs are

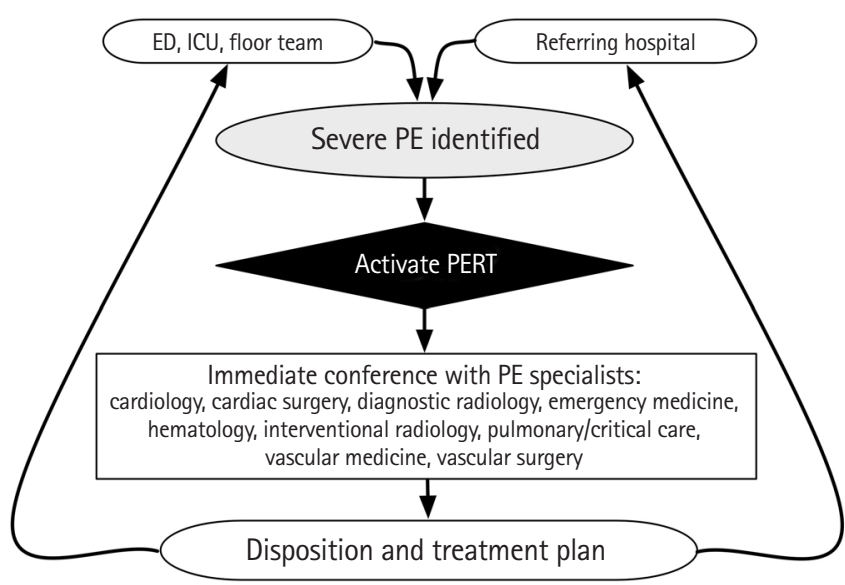

Fig. 1. Pulmonary embolism response team (PERT) approach. ED, emergency department; ICU, intensive care unit; $\mathrm{PE}$, pulmonary embolism.

designed to facilitate the efficient mobilization of resources and offer the benefit of multi-specialty expertise to patients with highand intermediate-risk PE. The PERT from Massachusetts General Hospital, where the PERT concept originated, recently reported their experience treating 394 patients with high and intermediate risk PE. The PERT paradigm may become the new standard of care for patients with PE. ${ }^{39,40}$ The PERT can be activated from any area of the hospital and in some cases prior to the arrival of transfer patients when severe PE is identified. As seen in Fig. 1, after activation there is a real-time meeting of a multidisciplinary team, which discusses appropriate treatment options and disposition and then makes their recommendations. The PERT activation mobilizes resources needed to make room in the intensive care unit, operating room, and/or angiography suite when emergent intervention is needed and greatly facilitates timely care of the sickest patients presenting with PE.

\section{CONCLUSION}

PE diagnosis and treatment remains challenging for emergency physicians. Symptoms can be vague or non-existent, and the clinical presentation shares features with many other common diagnoses. Diagnostic testing varies based on pretest probability and many testing modalities are not without their own risks. Recent advances in treatment have emerged as have multidisciplinary teams to assess and tailor treatment to individual patients needs.

\section{CONFLICT OF INTEREST}

No potential conflict of interest relevant to this article was reported. 


\section{REFERENCES}

1. Office of the Surgeon General. The surgeon general's call to action to prevent deep vein thrombosis and pulmonary embolism [Internet]. Rockville, MD: US Department of Health and Human Services; 2008 [cited 2016 Jun 6]. Available from: http://www.ncbi.nlm.nih.gov/books/NBK44178/.

2. Kucher N, Rossi E, De Rosa M, Goldhaber SZ. Massive pulmonary embolism. Circulation 2006;113:577-82.

3. Fanikos J, Piazza G, Zayaruzny M, Goldhaber SZ. Long-term complications of medical patients with hospital-acquired venous thromboembolism. Thromb Haemost 2009;102:688-93.

4. Singh $A$, editor. Scientific American medicine. Hamilton, ON: Decker Intellectual Properties; 2015.

5. Heit JA, Sobell JL, Li H, Sommer SS. The incidence of venous thromboembolism among Factor $\mathrm{V}$ Leiden carriers: a community-based cohort study. J Thromb Haemost 2005;3:305-11.

6. Poort SR, Rosendaal FR, Reitsma PH, Bertina RM. A common genetic variation in the 3 - -untranslated region of the prothrombin gene is associated with elevated plasma prothrombin levels and an increase in venous thrombosis. Blood 1996;88: 3698-703.

7. Ginsburg KS, Liang $M H_{1}$ Newcomer $L$, et al. Anticardiolipin antibodies and the risk for ischemic stroke and venous thrombosis. Ann Intern Med 1992;117:997-1002.

8. Goldhaber SZ, Grodstein F, Stampfer MJ, et al. A prospective study of risk factors for pulmonary embolism in women. JAMA 1997;277:642-5.

9. Prandoni P, Bilora F, Marchiori $A$, et al. An association between atherosclerosis and venous thrombosis. N Engl J Med 2003; 348:1435-41.

10. Spencer FA, Ginsberg JS, Chong A, Alter DA. The relationship between unprovoked venous thromboembolism, age, and acute myocardial infarction. J Thromb Haemost 2008;6:1507-13.

11. Stein PD, Beemath A, Meyers FA, Skaf E, Sanchez J, Olson RE. Incidence of venous thromboembolism in patients hospitalized with cancer. Am J Med 2006;119:60-8.

12. Petitti DB. Clinical practice: combination estrogen-progestin oral contraceptives. N Engl J Med 2003;349:1443-50.

13. Meng $K_{1}$ Hu $X$, Peng $X$, Zhang $Z$. Incidence of venous thromboembolism during pregnancy and the puerperium: a systematic review and meta-analysis. J Matern Fetal Neonatal Med 2015;28:245-53.

14. Beam DM, Courtney DM, Kabrhel C, Moore CL, Richman PB, Kline JA. Risk of thromboembolism varies, depending on category of immobility in outpatients. Ann Emerg Med 2009;54: $147-52$.
15. Laporte $S$, Mismetti $P$, Decousus $H$, et al. Clinical predictors for fatal pulmonary embolism in 15,520 patients with venous thromboembolism: findings from the Registro Informatizado de la Enfermedad TromboEmbolica venosa (RIETE) Registry. Circulation 2008;117:1711-6.

16. Kline JA, Mitchell AM, Kabrhel C, Richman PB, Courtney DM. Clinical criteria to prevent unnecessary diagnostic testing in emergency department patients with suspected pulmonary embolism. J Thromb Haemost 2004;2:1247-55.

17. Penaloza A, Verschuren F, Meyer G, et al. Comparison of the unstructured clinician gestalt, the wells score, and the revised Geneva score to estimate pretest probability for suspected pulmonary embolism. Ann Emerg Med 2013;62:117-124.e2.

18. Kline JA, Courtney DM, Kabrhel $C$, et al. Prospective multicenter evaluation of the pulmonary embolism rule-out criteria. J Thromb Haemost 2008;6:772-80.

19. Fesmire $F M$, Brown MD, Espinosa JA, et al. Critical issues in the evaluation and management of adult patients presenting to the emergency department with suspected pulmonary embolism. Ann Emerg Med 2011;57:628-652.e75.

20. Pomero $F$, Dentali $F$, Borretta $V$, et al. Accuracy of emergency physician-performed ultrasonography in the diagnosis of deepvein thrombosis: a systematic review and meta-analysis. Thromb Haemost 2013;109:137-45.

21. Duriseti RS, Brandeau ML. Cost-effectiveness of strategies for diagnosing pulmonary embolism among emergency department patients presenting with undifferentiated symptoms. Ann Emerg Med 2010;56:321-332.e10.

22. Kabrhel C, Mark Courtney D, Camargo CA Jr, et al. Potential impact of adjusting the threshold of the quantitative D-dimer based on pretest probability of acute pulmonary embolism. Acad Emerg Med 2009;16:325-32.

23. Righini M, Van Es J, Den Exter PL, et al. Age-adjusted D-dimer cutoff levels to rule out pulmonary embolism: the ADJUST-PE study. JAMA 2014;311:1117-24

24. Goodman LR, Stein PD, Matta F, et al. CT venography and compression sonography are diagnostically equivalent: data from PIOPED II. AJR Am J Roentgenol 2007;189:1071-6.

25. van Belle $A$, Buller HR, Huisman MV, et al. Effectiveness of managing suspected pulmonary embolism using an algorithm combining clinical probability, D-dimer testing, and computed tomography. JAMA 2006;295:172-9.

26. Kearon C, Akl EA, Ornelas J, et al. Antithrombotic therapy for VTE disease: CHEST guideline and expert panel report. Chest 2016;149:315-52.

27. Furlan A, Aghayev A, Chang CC, et al. Short-term mortality in acute pulmonary embolism: clot burden and signs of right 
heart dysfunction at CT pulmonary angiography. Radiology 2012;265:283-93.

28. Sanchez O, Trinquart L, Caille V, et al. Prognostic factors for pulmonary embolism: the prep study, a prospective multicenter cohort study. Am J Respir Crit Care Med 2010;181:168-73.

29. Piazza G, Goldhaber SZ. The acutely decompensated right ventricle: pathways for diagnosis and management. Chest 2005; 128:1836-52.

30. Becattini C, Vedovati MC, Agnelli G. Prognostic value of troponins in acute pulmonary embolism: a meta-analysis. Circulation 2007;116:427-33.

31. Kabrhel $C$, Okechukwu I, Hariharan $P$, et al. Factors associated with clinical deterioration shortly after PE. Thorax 2014;69: 835-42.

32. Chatterjee S, Chakraborty A, Weinberg I, et al. Thrombolysis for pulmonary embolism and risk of all-cause mortality, major bleeding, and intracranial hemorrhage: a meta-analysis. JAMA 2014;311:2414-21.

33. Lee $A Y$, Levine $M N$, Baker $\mathrm{RI}$, et al. Low-molecular-weight heparin versus a coumarin for the prevention of recurrent venous thromboembolism in patients with cancer. N Engl J Med 2003; 349:146-53.

34. Meyer $\mathrm{G}$, Vicaut $\mathrm{E}$, Danays $\mathrm{T}$, et al. Fibrinolysis for patients with intermediate-risk pulmonary embolism. N Engl J Med 2014; 370:1402-11.

35. Kline JA, Nordenholz KE, Courtney DM, et al. Treatment of submassive pulmonary embolism with tenecteplase or placebo: cardiopulmonary outcomes at 3 months: multicenter double-blind, placebo-controlled randomized trial. J Thromb Haemost 2014;12:459-68.

36. Kucher N, Boekstegers P, Muller OJ, et al. Randomized, controlled trial of ultrasound-assisted catheter-directed thrombolysis for acute intermediate-risk pulmonary embolism. Circulation 2014;129:479-86.

37. Kuo WT, Gould MK, Louie JD, Rosenberg JK, Sze DY, Hofmann LV. Catheter-directed therapy for the treatment of massive pulmonary embolism: systematic review and meta-analysis of modern techniques. J Vasc Interv Radiol 2009;20:1431-40.

38. Leacche $M$, Unic $D$, Goldhaber $S Z$, et al. Modern surgical treatment of massive pulmonary embolism: results in 47 consecutive patients after rapid diagnosis and aggressive surgical approach. J Thorac Cardiovasc Surg 2005;129:1018-23.

39. Provias T, Dudzinski DM, Jaff MR, et al. The Massachusetts General Hospital Pulmonary Embolism Response Team (MGH PERT): creation of a multidisciplinary program to improve care of patients with massive and submassive pulmonary embolism. Hosp Pract (1995) 2014;42:31-7.

40. Kabrhel C, Rosovsky R, Channick R, et al. A Multidisciplinary Pulmonary Embolism Response Team (PERT): initial 30-month experience with a novel approach to delivery of care to patients with sub-massive and massive PE. Chest 2016;150:38493. 